\title{
A deletion in the Ctns gene causes renal tubular dysfunction and cystine accumulation in LEA/Tohm rats
}

\author{
Yukiko Shimizu $^{1,2} \cdot$ Rieko Yanobu-Takanashi $^{1} \cdot$ Kenta Nakano $^{1,3} \cdot$ Kenji Hamase $^{4} \cdot$ Toshiaki Shimizu $^{2}$. \\ Tadashi Okamura ${ }^{1,5}$
}

Received: 4 November 2018 / Accepted: 19 December 2018 / Published online: 27 December 2018

(c) The Author(s) 2018

\begin{abstract}
The Long-Evans Agouti (LEA/Tohm) rat has recently been established as a new rat model of type 2 diabetes. The onset of diabetes mellitus was observed only in male LEA/Tohm rats; however, urinary glucose appeared before the onset of diabetes. To clarify the genetic basis of urinary glucose, we performed genetic linkage analysis using $(B N \times L E A) \mathrm{F}_{2}$ intercross progeny. A recessively acting locus responsible for urinary glucose excretion $(\mathrm{ugl})$ was mapped to a $7.9 \mathrm{Mb}$ region of chromosome 10, which contains the cystinosin (Ctns) gene. The Ctns gene encodes the cystine transporter, which transports cystine out of the lysosome and is responsible for nephropathic cystinosis in humans. Sequence analysis identified a 13-bp deletion in the Ctns gene, leading to a truncated and loss-of-function protein, which cause cystine accumulation in various tissues. We also developed a novel congenic rat strain harboring the Ctns $^{u g l}$ mutation on the F344 genetic background. Phenotypic analysis of F344-Ctns $s^{u g l}$ rats indicated that the incidence of urinary glucose was $100 \%$ in both males and females at around 40 weeks of age, and marked cystine accumulation was observed in the tissues, as well as remarkable renal lesions and cystine crystals in the lysosomes of the renal cortex. Furthermore, treatment with cysteamine depleted the cystine contents in F344-Ctns ${ }^{u g l}$ rat embryonic fibroblasts. These results indicated that the F344-Ctns ${ }^{u g l}$ rat provides a novel rat model of cystinosis, which allows not only a better understanding of the pathogenesis and pathophysiology of cystinosis but will also contribute to the development of new therapies.
\end{abstract}

Electronic supplementary material The online version of this article (https://doi.org/10.1007/s00335-018-9790-3) contains supplementary material, which is available to authorized users.

Tadashi Okamura

okamurat@ri.ncgm.go.jp

1 Department of Laboratory Animal Medicine, Research Institute, National Center for Global Health and Medicine (NCGM), 1-21-1 Toyama, Shinjyuku-ku, Tokyo 162-8655, Japan

2 Department of Pediatrics and Adolescent Medicine, Juntendo University Graduate School of Medicine, 2-1-1 Hongo, Bunkyo-ku, Tokyo 113-8421, Japan

3 Laboratory of Laboratory Animal Science and Medicine, School of Veterinary Medicine, Kitasato University, Higashi 23-35-1, Towada, Aomori 034-8628, Japan

4 Graduate School of Pharmaceutical Sciences, Kyushu University, 3-1-1 Maidashi, Higashi-ku, Fukuoka 812-8582, Japan

5 Section of Animal Models, Department of Infections Diseases, Research Institute, National Center for Global Health and Medicine (NCGM), 1-21-1 Toyama, Shinjyuku-ku, Tokyo 162-8655, Japan

\section{Introduction}

Cystinosis is a rare progressive lysosomal storage disorder characterized by the accumulation of cystine in lysosomes throughout every cell in the body. This results in various clinical characteristics such as Fanconi syndrome, poor growth, photophobia, hypothyroidism, end-stage renal disease (ESRD), hypothyroidism, hypogonadism, muscle weakness, swallowing difficulties, and pulmonary dysfunction. In untreated individuals, glomerular function gradually deteriorates, resulting in renal failure at approximately 10 years of age (Gahl et al. 2002; Gahl 1986). Cystinosis is an autosomal recessive disorder caused by mutations that disable the cystine transporter called cystinosin (CTNS) (Town et al. 1998; Touchman et al. 2000). The CTNS gene encodes a seven-transmembrane domain-containing, cystine $/ \mathrm{H}^{+}$symporter lysosomal protein, which has an indispensable role in the efflux of cystine from the lysosome (Kalatzis et al. 2001). In patients with cystinosis, cystine (which is the oxidized dimer form of cysteine) accumulates continuously in the lysosome owing to the 
defective CTNS, resulting in the formation of cystine crystals in every cell in the body, leading to multi-organ damage. It is estimated that the frequency of cystinosis is 1 in 100,000-200,000 live births (Elmonem et al. 2016; Gahl et al. 2002); however, the incidence in East Asia is much lower (Chuang et al. 2012; Higashi et al. 2017; Yang et al. 2015) because the disease is often undiagnosed or misdiagnosed.

Cystinosis is one of the few rare diseases that have specific treatments. Oral cysteamine treatment, which depletes lysosomal cystine in all body cells and tissues, improves the prognosis of cystinosis by delaying the progression to ESRD (Brodin-Sartorius et al. 2012). Oral cysteamine therapy is effective in extending life expectancy and patient survival, but cannot cure the disease completely (Cherqui 2012; Gahl et al. 2007). However, new therapeutic approaches such as bone marrow and hematopoietic stem cell transplants (Gaide Chevronnay et al. 2016; Syres et al. 2009; Yeagy et al. 2011) are expected to offer a cure for cystinosis. Ctns knockout mice are thus useful for the development of new therapies, and to reveal the pathogenesis of cystinosis (Nevo et al. 2010; Prencipe et al. 2014).

Large amounts of urine with a strong odor, a possible indicator of diabetes mellitus, were frequently observed, and urinary glucose was detected in both male and female LEA/Tohm rats. We have previously reported a novel rat model of non-obese type 2 diabetes, the Long-Evans Agouti (LEA/Tohm) rat, which is characterized by a spontaneous insulin secretion-deficient diabetes model (Okamura et al. 2013). Spontaneous diabetes mellitus, as determined by oral glucose tolerance, was observed only in male LEA/Tohm rats, with the incidence of approximately $90 \%$ at 14 months of age, whereas none of the females developed diabetes. However, renal glucosuria appeared before the onset of diabetes in male rats and was present in all males and females at 9 months of age. Furthermore, the deterioration of renal tubular cells was evident in the proximal tubules at 12 months of age; in contrast, no proliferation of glomerulosclerosis or mesangial cells characteristic of diabetic nephropathy was observed, suggesting that renal glucosuria was not associated with the onset of diabetes. Therefore, we speculated that the renal tubular epithelial cells failed to reabsorb glucose, resulting in the development of renal glucosuria in LEA/Tohm rats and that diabetes mellitus observed in males is not correlated with renal glucosuria. In the present study, we identified the gene mutation responsible for glucosuria in LEA/ Tohm rats (designated $C t n s^{u g l}$ ) using a genetic association study and a candidate gene approach. We also established a novel congenic strain harboring the $C t n s^{u g l}$ mutation derived from the LEA/Tohm rat on a F344 standard rat strain, with the goal of establishing a novel rat model to be used for the further study of cystinosis.

\section{Materials and methods}

\section{Animals}

The LEA/Thom rats were obtained from the Institute for Animal Experimentation, Tohoku University Graduate School of Medicine, and were maintained at the Research Institute at the National Center for Global Health and Medicine (NCGM). BN/ssNSlc and F344/NSlc rats were purchased from Japan SLC (Hamamatsu, Japan). All rats were housed in an air-conditioned animal room at $23 \pm 2{ }^{\circ} \mathrm{C}$ and relative humidity of $40-60 \%$ under specific-pathogen-free (SPF) conditions, with a 12-h light/dark cycle (8:00-20:00/20:00-8:00). All rats were fed a standard rodent CE-2 diet (CLEA Japan, Tokyo, Japan) and had ad libitum access to water.

\section{Genetic mapping}

For genetic mapping of the $u g l$ mutant locus, $\mathrm{F}_{1}$ progeny were generated by crossing LEA/Tohm rats with $\mathrm{BN}$ rats, which were then used to produce the $\mathrm{F}_{2}$ intercross progeny. Genomic DNA samples from $47 \mathrm{~F}_{2}$ intercross progeny were genotyped using polymorphic 201 microsatellite markers (hereinafter referred to as SSLP markers, Supplemental Table S1). Genotyping was carried out via PCR and 4\% agarose electrophoresis. A total of $196 \mathrm{~F}_{2}$ intercross progeny were genotyped for the $u g l$ locus as per the presence or absence of urine glucose. Eleven SSLP markers (including an additional five markers) located on rat chromosome 10 were used for fine mapping (Supplemental Table S1). The association between the $u g l$ locus and each SSLP marker were individually evaluated via $\chi^{2}$ test using JMP7 statistics software (SAS Institute Japan, Tokyo, Japan) (Masuyama et al. 2003).

\section{Identification of the Ctns mutation}

The total RNAs were extracted from the kidneys of LEA/ Tohm and BN rats using an ISOGEN RNA extraction kit (Nippon Gene, Tokyo, Japan) according to the manufacturer's instructions. The total RNA was made to react with random hexamer primers and ReverTra Ace reverse transcriptase (Toyobo, Osaka, Japan). PCR analyses were performed on cDNA using primer pairs (5'-AGTGATAGGTGG AGAGGACAGAA-3'; 5'-ACCAGGCCATGAAGTAGA-3' and 5'-GGTCTCTCTGCTCCTCCC-3'; 5'-TCGTACCCT GGTTTCTTTCTGTA-3') for amplifying the entire coding regions of the Ctns gene. Sequence analysis was performed with an ABI PRISM 3100 Genetic Analyzer (Applied 
Biosystems, Foster City, CA, USA) and GENETYX-MAC Ver .13 (GENETYX, Tokyo, Japan).

\section{Development of congenic strain}

We created a congenic strain using marker-assisted selection protocols (Markel et al. 1997), the so-called "speed congenic" methods, for reducing the number of backcross generations needed to establish the congenic strain. The individual with the closest F344 strain type was selected from each generation using microsatellite genotyping, which was carried out via PCR with SSLP markers (Supplemental Table S2). To introgress the $C_{\text {tns }}{ }^{\text {ugl }}$ mutation from LEA/ Tohm into the inbred F344 strain (F344-Ctns ${ }^{u g l}$ ), the $\mathrm{F}_{1}$ (F344 $\times$ LEA/Tohm) progeny heterozygous for $\mathrm{Ctns}^{\text {ugl }}$ mutation were backcrossed to F344 rats for seven generations, and homozygous F344-Ctns ${ }^{\text {ugl }}$ rats were subsequently produced and maintained by sib-mating. F344-Ctns ${ }^{u g l}$ rats deposited to the National BioResource Project-Rat (NBRP-Rat).

\section{RT-qPCR analysis}

RT-qPCR analyses were performed as previously described, with minor modifications (Nakano et al. 2018). Briefly, total RNAs from the kidneys and livers of 14-week-old male BN and LEA/Tohm rats were extracted as described above, and cDNA was prepared with ReverTra Ace (Toyobo). For quantification, Ctns gene-specific primers (5'-TGGATCTACTTC ATGGCCTGGTC-3' and 5'-TAAGCTGAAGAAGGCGTC GTT-3') were used with THUNDERBIRD SYBR qPCR Mix (Toyobo) in a 7900HT Fast Real-Time PCR System (Applied Biosystems). The expression level of the Ctns gene was normalized to the level of Actb mRNA amplified by specific primers (5'-CGCATCCTCTTCCTCCCT- $3^{\prime}$ and 5'-CAGACAGCACTGTGTTGGCAT-3'). Each experiment was performed in three biological replicates.

\section{Measurement of urine glucose}

Urine samples from 16-h-fasted rats were collected by gentle manual compression of the abdomen. Urine glucose levels were measured semiquantitatively using uropaper AG2 (Eiken, Tokyo, Japan). Glucosuria is defined as a urine glucose level of $50 \mathrm{mg} / \mathrm{dL}$ or higher.

\section{Measurement of tissue cystine contents}

Rat tissues were homogenized in $500 \mu \mathrm{L}$ of $0.9 \%$ saline and were then deproteinized in $3 \mathrm{~mL}$ of $5 \%$ trichloroacetic acid solution with an internal standard (L-norvaline, Wako Pure Chemical Industries, Ltd., Osaka, Japan). After adding an equivalent volume of hexane, the homogenates were mixed and centrifuged for $15 \mathrm{~min}$ at $1600 \times g$ at room temperature and the aqueous portion (lower phase) was collected. For sensitive detection, cystine molecules were derivatized with 4-fluoro-7-nitro-2,1,3-benzoxadiazole (NBD-F) (Hamase 2007). To $5 \mu \mathrm{L}$ of the aqueous solution, $5 \mu \mathrm{L}$ of $\mathrm{H}_{2} \mathrm{O}, 10 \mu \mathrm{L}$ of $400 \mathrm{mM} \mathrm{Na}$-borate buffer ( $\mathrm{pH} 8.0$ ), and $5 \mu \mathrm{L}$ of $40 \mathrm{mM}$ NBD-F (Tokyo Kasei Kogyo, Tokyo, Japan) in acetonitrile solution (Nacalai Tesque, Kyoto, Japan) were added, and the solution was heated at $60^{\circ} \mathrm{C}$ for $2 \mathrm{~min}$. After adding $75 \mu \mathrm{L}$ of $2 \%$ trifluoroacetic acid (TFA) to terminate the reaction, an aliquot of this reaction mixture $(2 \mu \mathrm{L})$ was subjected to high-performance liquid chromatography (HPLC, Shimadzu Corporation, Kyoto, Japan) with Nucleonavi column (1.0 mm I.D $\times 250 \mathrm{~mm}$; Shiseido, Tokyo, Japan), to quantify the cystine-NBD derivatives. The mobile phase was $22.5 \%$ $\mathrm{v} / \mathrm{v}$ acetonitrile and $0.05 \% \mathrm{v} / \mathrm{v}$ TFA in $\mathrm{H}_{2} \mathrm{O}$ at the flow rate of $100 \mu \mathrm{L} / \mathrm{min}$, and fluorescence detection was carried out at $530 \mathrm{~nm}$ with excitation at $470 \mathrm{~nm}$.

\section{Histopathological analysis}

Rats were anesthetized using sevoflurane and were perfused intracardially with PBS followed by $10 \%$ neutral buffered formalin (Nacalai Tesque). The kidneys were removed and embedded in paraffin according to routine procedures. Paraffin sections were sliced at a thickness of $3 \mu \mathrm{m}$ and stained with hematoxylin and eosin (HE). Masson's trichrome staining was also conducted to assess fibrosis in the kidney. The stained sections were captured using optical microscopy (BX-51; Olympus, Tokyo, Japan).

For electron microscopy, the kidneys were fixed in $2.5 \%$ glutaraldehyde/PBS solution for $2 \mathrm{~h}$ at room temperature. Fixed tissues were washed with Milli Q water three times and stained en bloc with $0.5 \%$ uranyl acetate for $2 \mathrm{~h}$, dehydrated in an ethanol series, and then embedded in Quetol 812 (Nisshin EM, Tokyo, Japan). The blocks were trimmed to an area of approximately $0.5 \mathrm{~mm}^{2}$ and serially sectioned at a thickness of $100 \mathrm{~nm}$ with an ultramicrotome (Leica EM UC7, Leica, Vienna, Austria). The thin sections were then examined using an electron microscope (JEM-1400, JEOL, Tokyo, Japan).

\section{Preparation of rat embryonic fibroblast cells and drug assay}

Pregnant F344-Ctns ${ }^{\text {ugl/ugl }}$ rats at 15 d.p.c (day post-coitum) were euthanized via sevoflurane overdose. The uterine horns were dissected out, briefly rinsed in $70 \%(\mathrm{v} / \mathrm{v})$ ethanol, and washed with PBS. Each embryo was separated from its placenta and embryonic sac, and the head and red organs were removed from isolated embryos. The remaining bodies were washed with PBS and minced with scissors until they are possible to pipette. The tissues were transferred into $0.1 \mathrm{mM}$ trypsin/1 mM EDTA solution (Nacalai Tesque), and 
incubated at $37^{\circ} \mathrm{C}$ with mixing by inversion for $20 \mathrm{~min}$. The suspension was filtered using a cell strainer (BD biosciences, Bedford, MA, USA), followed by centrifugation for $5 \mathrm{~min}$ at $160 \times g$. The pellets were dissociated by gentle pipetting in REF culture medium (DMEM containing 10\% fetal bovine serum). The P0 (passage 0) fibroblasts were grown in REF medium until $\mathrm{P} 8$ in $5 \% \mathrm{CO}_{2}: 95 \%$ (v/v) air, and used for drug analyses. The cells were incubated in REF medium containing cysteamine, bucillamine (Wako Pure Chemical Industries, Ltd.), or captopril (Sigma-Aldrich, St. Louis, MO, USA) for $24 \mathrm{~h}$. The cystine contents of the drug-treated cells were determined via HPLC as described above.

\section{Statistics}

Data were presented as mean \pm standard error of the mean $(\mathrm{SEM})$. Data were analyzed using Student's $t$ test. Correction of multiple comparisons was calculated using Bonferroni correction. Statistical analysis was performed using Excel Statistic and JMP (ver.7.0.1) software. A $P$ value $<0.05$ was considered statistically significant.

\section{Results}

\section{Linkage analysis and identification of the ugl mutation}

Though only male LEA/Tohm rats developed diabetes mellitus with the incidence of $90 \%$ at 14 months of age, the urinary glucose was positive before the onset of diabetes at 9 months (approximately 40 weeks) of age (Okamura et al. 2013). To determine the mode of inheritance of renal glycosuria in LEA/Tohm rats, we generated $(\mathrm{BN} \times \mathrm{LEA} / \mathrm{Tohm})$ $\mathrm{F}_{2}$ intercross progeny. Of the 196 male offspring, 52 (26.5\%) and $144(73.5 \%)$ individuals at 52 weeks of age were positive and negative for urinary glucose, respectively, indicating that renal glycosuria in LEA/Tohm rats is inherited in a recessive fashion. Next, we genotyped DNA samples from the 47 male $\mathrm{F}_{2}$ intercross progeny using 201 SSLP markers (Supplemental Table S1) on rat chromosomes 1-20 and X to determine the urinary glucose excretion ( $u g l$ locus. Using $\chi^{2}$ analysis, we detected significant association of the $u g l$ locus with SSLP markers on chromosomes 2, 8, 10, and 16 and mapped the $u g l$ locus to an interval of approximately $45 \mathrm{Mb}$ between D10Rat34 $(P=2.2 \mathrm{E}-5)$ and D10Rat151 $(P=5.85 \mathrm{E}-7)$ around D10Mgh6 $(P=7.1 \mathrm{E}-12)($ Table 1$)$. By using the male $196 \mathrm{~F}_{2}$ intercross progeny and examining an additional five SSLP markers on chromosome 10 (Supplemental Table S1), the haplotype analysis revealed that the $u g l$ locus was located within an interval of $7.9 \mathrm{Mb}$ between D10Rat77 $(P=3.16 \mathrm{E}-41)$ and D10Mgh6 $(P=1.96 \mathrm{E}-43)$ (Table 2, Supplemental Fig. S1). No recombination could be seen between the ugl, DlORat241 and DloRat80 $(P=1.56 \mathrm{E}-44)$. This region contains 132 protein-coding genes, including the $u g l$ candidate region [The Rat Genome Database (RGD) (https://rgd.mcw.edu)]. Given the information regarding the candidate genes for this locus based on RGD, Ctns was the strongest candidate gene for the $u g l$ locus. By analyzing the sequence of $C t n s$, which consists of
Table 1 SSLP markers showing significant associations between genotype and urine glucose positivity on $47 \mathrm{~F}_{2}$ intercross progeny

\begin{tabular}{|c|c|c|c|c|c|c|c|c|}
\hline \multirow[t]{2}{*}{ Chr. } & \multirow[t]{2}{*}{ Locus name } & \multirow[t]{2}{*}{ Position $(\mathrm{Mb})^{\mathrm{a}}$} & \multicolumn{2}{|c|}{$\begin{array}{l}\text { Urine glucose-nega- } \\
\text { tive }^{\text {b }}\end{array}$} & \multicolumn{2}{|c|}{ Urine glucose-positive $^{c}$} & \multirow[t]{2}{*}{$\chi^{2}$} & \multirow[t]{2}{*}{$P$ value } \\
\hline & & & $\begin{array}{l}\text { BN/BN or } \\
\text { BN/LEA }\end{array}$ & LEA/LEA & $\begin{array}{l}\mathrm{BN} / \mathrm{BN} \text { or } \\
\mathrm{BN} / \mathrm{LEA}\end{array}$ & LEA/LEA & & \\
\hline 2 & D2Rat112 & 249.3 & 27 & 8 & 5 & 7 & 5.17 & 0.02 \\
\hline 8 & D8Rat47 & 39.3 & 30 & 5 & 7 & 5 & 4.00 & 0.05 \\
\hline \multirow[t]{7}{*}{10} & D10Rat121 & 16.8 & 27 & 8 & 5 & 7 & 5.18 & 0.02 \\
\hline & D10Rat181 & 17.7 & 28 & 7 & 5 & 7 & 6.28 & 0.01 \\
\hline & D10Rat42 & 21.7 & 28 & 7 & 5 & 7 & 6.28 & 0.01 \\
\hline & D10Rat34 & 32.5 & 31 & 4 & 3 & 9 & 18.1 & $2.20 \mathrm{E}-05$ \\
\hline & D10Mgh6 & 64.6 & 35 & 0 & 0 & 12 & 47.0 & $7.10 \mathrm{E}-12$ \\
\hline & D10Rat151 & 77.5 & 32 & 3 & 2 & 10 & 25.0 & $5.85 \mathrm{E}-07$ \\
\hline & D10Rat122 & 91.3 & 29 & 6 & 4 & 8 & 10.5 & $1.20 \mathrm{E}-03$ \\
\hline \multirow[t]{2}{*}{16} & D16Rat34 & 73.0 & 25 & 10 & 12 & 0 & 4.36 & 0.04 \\
\hline & D16Rat37 & 75.8 & 23 & 12 & 12 & 0 & 5.52 & 0.02 \\
\hline
\end{tabular}

$\chi^{2}$ test $P$ value lower than 0.05 are shown. Whole genome scan was performed in $47 \mathrm{~F}_{2}$ progeny using 201 SSLP markers as shown in Supplemental Table S1

${ }^{a}$ Rattus norvegicus (Norway rat) genome assembly Rnor_6.0

${ }^{b} \mathrm{~F}_{2}$ rats having urine glucose lower than $50 \mathrm{mg} / \mathrm{dL}$ under fasting conditions

${ }^{\mathrm{c}} \mathrm{F}_{2}$ rats having urine glucose higher than $50 \mathrm{mg} / \mathrm{dL}$ under fasting conditions 
10 coding exons and encodes 367 amino acids, we identified a 13-bp deletion at position 525 (c.525_537del) in exon 7 of Ctns in LEA/Tohm rats (Fig. 1a), causing a frame shift mutation at codon 177 with subsequent termination after an additional 17 codons. We designed genotyping PCR primers to detect the 13-bp deletion in the Ctns gene, and examined the variation in exon 7 of $C$ tns among the LEA substrains. The 13-bp deletion was observed in LEA/Tohm and LEA/ Hkm, but not in LEA/Tj (Nakajima et al. 1994) and LEA/ Hok rats (Agui et al. 2001) (Supplemental Fig. S2).

Next, we examined the mRNA expression of the Ctns gene in the liver and kidneys from $\mathrm{BN}$ and LEA/Tohm rats via quantitative RT-PCR. The relative abundance of $C t n s$ transcripts from LEA/Tohm rats is markedly lower in the liver (16\%) and kidney (10\%) (Fig. 1b), which may be caused by nonsense-mediated mRNA decay (NMD), resulting in systemic cystine accumulation in all tissues. Therefore, the intracellular cystine content of different organs from LEA/ Tohm and BN rats was assayed via HPLC. Cystine content was significantly increased in the liver, eyes, heart and spleen of LEA/Tohm rats at 8-14 weeks of age in comparison to that of BN rats (Table 3). At 52 weeks of age, cystine accumulation was clearly observed in all organs tested, with the highest levels in the spleen (430-fold) and the lowest in the brain (1.8-fold) compared with age-matched BN rats. An increase of 12.3-fold was seen in the liver, 3.9-fold in the kidneys, 83-fold in the heart, 9.2-fold in the lungs, 36-fold in the muscles, and 90-fold in the eyes (Table 3), and these accumulations increased with age. Cystine crystal structures, which are one of the typical pathological findings in cystinosis patients (Gahl et al. 2002) and mouse models of cystinosis (Cherqui 2002; Nevo et al. 2010), were present in the kidney cortex (Fig. 1c). These results indicate that renal glycosuria in the LEA/Tohm rat was caused by a deletion of 13-bp in Ctns.

\section{Quantitative Trait Loci (QTL) analysis for kidney function}

To examine whether glucosuria was correlated with renal function in LEA/Tohm rats, we performed QTL analysis for serum creatinine using the same $\mathrm{F}_{2}$ intercross progeny as mentioned above (Supplemental Fig. S3). We identified one significant QTL peak on chromosome 10 at D10Mgh6 $(64.6 \mathrm{Mb})$ with a likelihood ratio statistic (LRS) score of 64.1, and one suggestive QTL peak on chromosome 12 at D12Rat23 (27.2 Mb) with an LRS score of 12.8 (Supplemental Fig. S4a). D10Mgh6 is located close to the $u g l$ locus, which is responsible for urinary glucose excretion (Supplemental Fig. S4b) strongly indicating a correlation between the two phenotypes.

\section{Development of a novel rat model for cystinosis}

The LEA rats were established from an outbred colony of Long-Evans rats, and were sometimes used as a control strain for the Long-Evans Cinnamon (LEC) rats, a rat model for Wilson's disease, since they do not have the Atp7b mutation (Sasaki et al. 1994). However, LEA rats carry various other mutations such as impaired glucose tolerance (Okamura et al. 2013), X-ray hypersensitivity (Agui et al. 2001), and lack of D-amino acid oxidase activity (Konno et al. 2009), and are, therefore, not necessarily suitable as a cystinosis model. We established a congenic rat line in
Table $2 \chi^{2}$ test for association between genotype on rat chromosome 10 and urine glucose positivity using $196 \mathrm{~F}_{2}$ intercross progeny

\begin{tabular}{|c|c|c|c|c|c|c|c|}
\hline \multirow[t]{2}{*}{ Locus name } & \multirow[t]{2}{*}{ Position $(\mathrm{Mb})^{\mathrm{a}}$} & \multicolumn{2}{|c|}{ Urine glucose-negative $^{b}$} & \multicolumn{2}{|c|}{ Urine glucose-positive $^{c}$} & \multirow[t]{2}{*}{$\chi^{2}$} & \multirow[t]{2}{*}{$P$ value } \\
\hline & & $\begin{array}{l}\text { BN/BN or } \\
\text { BN/LEA }\end{array}$ & LEA/LEA & $\begin{array}{l}\mathrm{BN} / \mathrm{BN} \text { or } \\
\mathrm{BN} / \mathrm{LEA}\end{array}$ & LEA/LEA & & \\
\hline D10Rat95 & 6.1 & 110 & 34 & 36 & 16 & 1.03 & 0.31 \\
\hline D10Rat42 & 21.7 & 122 & 22 & 27 & 25 & 22.5 & $2.05 \mathrm{E}-06$ \\
\hline D10Rat34 & 32.5 & 131 & 13 & 16 & 36 & 73.8 & $8.43 \mathrm{E}-18$ \\
\hline D10Rat223 & 42.8 & 134 & 10 & 10 & 42 & 106.8 & $4.87 \mathrm{E}-25$ \\
\hline D10Rat77 & 56.7 & 143 & 1 & 2 & 50 & 180.90 & $3.16 \mathrm{E}-41$ \\
\hline D10Rat 241 & 59.7 & 144 & 0 & 0 & 52 & 196.0 & $1.56 \mathrm{E}-44$ \\
\hline ugl, Ctns & $59.75-59.77$ & & & & & & \\
\hline D10Rat80 & 61.3 & 144 & 0 & 0 & 52 & 196.0 & $1.56 \mathrm{E}-44$ \\
\hline D10Mgh6 & 64.6 & 143 & 1 & 0 & 52 & 191.0 & $1.96 \mathrm{E}-43$ \\
\hline D10Rat133 & 64.8 & 141 & 3 & 1 & 51 & 176.4 & $3.01 \mathrm{E}-40$ \\
\hline D10Rat151 & 77.5 & 136 & 8 & 13 & 39 & 101.10 & $8.89 \mathrm{E}-24$ \\
\hline D10Rat7 & 104.3 & 118 & 26 & 36 & 16 & 3.67 & 0.06 \\
\hline
\end{tabular}

${ }^{\mathrm{a}}$ Rattus norvegicus (Norway rat) genome assembly Rnor_6.0

${ }^{\mathrm{b}} \mathrm{F}_{2}$ rats having urine glucose lower than $50 \mathrm{mg} / \mathrm{dL}$ under fasting conditions

${ }^{\mathrm{c}} \mathrm{F}_{2}$ rats having urine glucose higher than $50 \mathrm{mg} / \mathrm{dL}$ under fasting conditions 
a
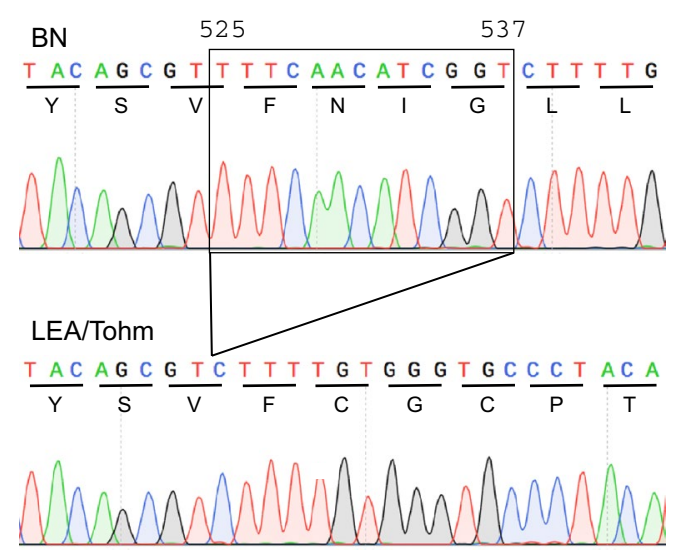

170

177

BN : FVAYSVFNIGLLWVPYIQEEFLLKY

LEA/TOhm: FVAYSVFCGCPTSRRSFSSNTPMV*

\section{b}

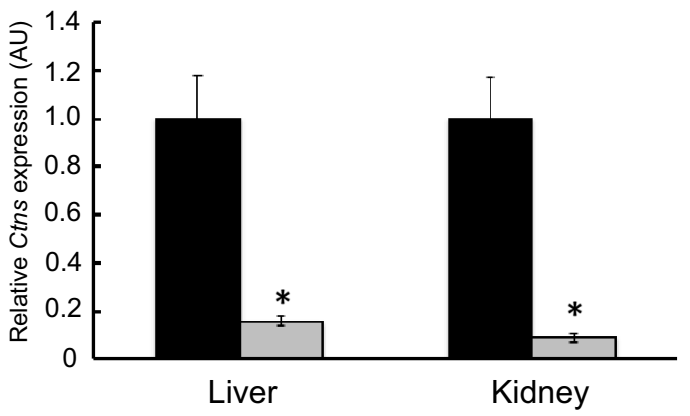

C

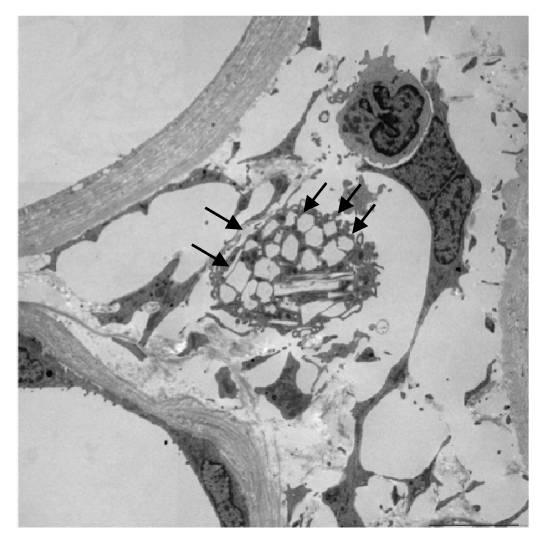

Fig. 1 Mutation analysis of Ctns in LEA/Tohm rats. a Partial electropherogram of Sanger sequencing of the Ctns cDNA from BN (+/+, upper panel) and LEA/Tohm (ugl/ugl, middle panel) rats. The reading frame is underlined. A 13-bp deletion (c.525_537del) causes a frame shift mutation at codon 177 , with subsequent termination after additional 17 codons (bottom panel). The stop codon is shown as an asterisk. b RT-qPCR analysis of the Ctns gene in LEA/Tohm liver and kidneys at 14 weeks of age. The values were arbitrary units after normalization against Actb. Data were analyzed using Student's $t$ test $\left({ }^{*} P<0.01\right)$. BN (closed bars; $\left.n=3\right)$ and LEA/Tohm (gray bars; $n=3$ ) rat. Each experiment was performed in three biological replicates. c A representative transmission electron microscopy (TEM) image of the LEA/Tohm kidney cortex at 52 weeks of age. Arrows indicate cystine crystals in macrophages of the renal interstitium. Magnification $\times 2500$

Table 3 Intracellular cystine levels in various tissues of LEA/Tohm rats

\begin{tabular}{|c|c|c|c|c|c|c|c|c|}
\hline & Liver & Kidney & Heart & Lung & Muscle & Brain & Eye & Spleen \\
\hline \multicolumn{9}{|l|}{ 8-14 weeks } \\
\hline $\mathrm{BN}(n=5)$ & $0.12 \pm 0.02$ & $1.25 \pm 0.07$ & $0.21 \pm 0.03$ & $0.34 \pm 0.02$ & $0.02 \pm 0.00$ & $0.26 \pm 0.02$ & $0.04 \pm 0.00$ & $0.08 \pm 0.01$ \\
\hline LEA $(n=5)$ & $0.34 \pm 0.07 *$ & $1.50 \pm 0.26$ & $0.47 \pm 0.10^{*}$ & $0.39 \pm 0.03$ & $0.10 \pm 0.03$ & $0.28 \pm 0.05$ & $0.12 \pm 0.03^{*}$ & $5.18 \pm 2.48^{*}$ \\
\hline \multicolumn{9}{|l|}{52 weeks } \\
\hline $\mathrm{BN}(n=3)$ & $0.10 \pm 0.03$ & $1.45 \pm 0.10$ & $0.07 \pm 0.03$ & $0.29 \pm 0.03$ & $0.01 \pm 0.00$ & $0.13 \pm 0.05$ & $0.02 \pm 0.00$ & $0.08 \pm 0.01$ \\
\hline LEA $(n=3)$ & $1.23 \pm 0.02 * *$ & $5.60 \pm 1.67 *$ & $5.81 \pm 0.45^{* *}$ & $2.66 \pm 0.18^{* *}$ & $0.36 \pm 0.06^{* *}$ & $0.23 \pm 0.05$ & $1.79 \pm 0.22 * *$ & $34.34 \pm 0.87 * *$ \\
\hline
\end{tabular}

Tissue cystine contents were measured as micromoles of half-cystine per gram of wet tissue, mean \pm SEM

$* P<0.05 ; * * P<0.01$ versus age-matched control $\mathrm{BN}$ rats

which the $C$ tns ${ }^{u g l}$ mutation was introduced into F344 rats using speed congenic methods (Supplemental Table S2). Glucosuria appeared at 28 weeks of age in the homozygous male F344-Ctns ${ }^{u g l}$ rats, and was present in $100 \%$ of males at 36 weeks of age. In the homozygous female rats, glucosuria appeared at 30 weeks of age and was present in $100 \%$ of females at 38 weeks of age (Fig. 2a). As seen in the kidneys of LEA/Tohm rats (Okamura et al. 2013), pathological analysis of F344-Ctns ${ }^{\text {ugl } / \text { ugl }}$ kidneys at 52 weeks of age revealed markedly expanded tubular lumen in the cortex as well as proximal tubular atrophy, flattened epithelium, and basement membrane thickening associated with the disappearance of the epithelial cell layer (Fig. 2b). Electron microscopy of the kidney cortex showed that a large 
number of cystine crystal structures were also present in intracellular lysosomes (Fig. 2b), as also seen in LEA/Tohm kidneys (Fig. 1c). Cystine content was markedly increased in all F344-Ctns ${ }^{\text {ugllugl }}$ rat tissues tested at 52 weeks of age. An increase of 4.3-fold was seen in the kidney, 154-fold in the spleen, and 117-fold in the bone marrow (Fig. 2c). Despite this, the F344-Ctns ${ }^{u g l / u g l}$ rat demonstrated normal growth and fertility. In addition, F344-Ctns ${ }^{\text {ugllugl }}$ rats had normal glycemic response during oral glucose tolerance test (Supplemental Fig. S5), indicating that $u g l$ locus on chromosome 10 is not associated with impaired glucose tolerance.

Furthermore, we established rat embryonic fibroblasts (REFs) from F344-Ctns ${ }^{\text {ugl }}{ }^{\text {ugl }}$ rats to test the effectiveness of cystine depletion by cysteamine, the only therapeutic drug for cystinosis, and evaluated whether the F344-Ctns ${ }^{\text {ugllugl }}$ rat was useful as a cystinosis model. Fibroblasts are frequently used in the study of cystinosis, especially in functional assessments (Iglesias et al. 2012; Sansanwal et al. 2010; Vitvitsky et al. 2010). The effect of drugs was evaluated based on cystine accumulation in REFs. The treatment with cysteamine resulted in a significant reduction of cystine content in a dose-dependent manner. A decrease of $22 \%$ was seen at $0.01 \mathrm{mM}(P=0.002), 53 \%$ at $0.05 \mathrm{mM}, 74 \%$ at $0.1 \mathrm{mM}$, and $>99.8 \%$ at $1 \mathrm{mM}$ cysteamine compared with the control (Fig. 3a). To show the availability of F344Ctns ${ }^{\text {ugl/ugl }}$ REFs in evaluating candidate drugs of cystinosis, we tested bucillamine and captopril, both of which are thiol compounds, which also decreased cystine accumulation in a dose-dependent manner. At a dose of $1 \mathrm{mM}$, bucillamine (93\% decrease) more effectively reduced the intracellular cystine levels than captopril (18\% decrease) (Fig. 3b). These results suggest that REFs from the F344-Ctns ${ }^{\text {ugl } / u g l}$ rat may be used for evaluation of candidate drugs of cystinosis.

\section{Discussion}

In the present study, we present several lines of evidence demonstrating that a mutation in the Ctns gene underlies spontaneous renal glucosuria in LEA/Tohm rats.

The mating test and linkage analysis using SSLP markers on the whole genome revealed that $u g l$ was inherited as an autosomal recessive trait and mapped to the region on chromosome 10 harboring the Ctns gene (Table 2 and Supplemental Fig. S1). The Ctns gene encodes a seven-transmembrane domain-containing protein that functions to transport cystine out of lysosomes, and it has been reported that nephropathic cystinosis is caused by CTNS gene mutations in humans (Attard et al. 1999; Kalatzis et al. 2004; Town et al. 1998). Cystinosis is a disease that causes impaired export of cystine from the lysosome to the cytoplasm, resulting in the accumulation of cystine in all organs, which leads to multi-organ damage and associated serious renal tubule symptoms (Elmonem et al. 2016; Gahl et al. 2002). Systemic accumulation of cystine was also observed in various tissues from LEA/Tohm rats (Table 3). Although there were 132 genes in the $u g l$ candidate region on chromosome 10 (Table 2, Supplemental Fig. S1), the Ctns gene was a strong candidate for the $u g l$ mutation. The 13-bp deletion in the Ctns gene is predicted to cause a frameshift mutation, resulting in truncation of the peptide chain owing to a stop codon generated at amino acid position 194. In silico prediction for the mutated protein was based on the system SOSUI; classification and secondary structure prediction of membrane proteins suggested that the truncated protein yields a soluble protein (Hirokawa et al. 1998). The Ctns frameshift mutation is supposed to completely disrupt the function of the protein, which, because of this frameshift, is not localized to lysosomes and has lost its cystine transport function. In addition to this, the marked accumulation of cystine in REFs from the F344-Ctns ${ }^{\text {ugl/ugl }}$ rat was depleted after treatment with cysteamine, a therapeutic agent for cystinosis. We therefore conclude that the $u g l$ mutation, which caused the degeneration of renal tubular epithelial cells, development of glucosuria, and cystine accumulation in LEA/Tohm rats, is the 13-bp deletion in the Ctns gene.

F344-Ctns $s^{\text {ugl } / u g l}$ rats carrying the $C$ tns ${ }^{\text {ugl }}$ mutation in the F344 genetic background exhibited renal proximal tubulopathy, and incidence of glucosuria reached $100 \%$ in both males and females at around 40 weeks of age. The onset of glucosuria in F344-Ctns $s^{\text {ug/ugll }}$ rats was slightly delayed compared to that in LEA/Tohm rats (Okamura et al. 2013) (Fig. 2a). Reportedly, renal dysfunction in $\mathrm{Ctns}^{-/-}$mice was dependent on their genetic background (Nevo et al. 2010). $\mathrm{Ctns}^{-1-}$ mice on the C57BL/6 genetic background developed focal lesions affecting the proximal tubules at 6 months of age, with more extensive lesion development at 9 months, whereas the $\mathrm{Ctns}^{-/-}$mice having FVB/N and a mixed $129 \mathrm{~Sv} \times \mathrm{C} 57 \mathrm{BL} / 6$ genetic backgrounds present no signs of proximal tubulopathy, even at 18 months of age. The reason for these strain differences could be explained by the amount of cystine accumulation in the kidney in each congenic strain. The renal cystine levels of C57BL/6 $\mathrm{Ctns}^{-1-}$ mice were much higher than those of FVB/N and the mixed $129 \mathrm{~Sv} \mathrm{Ctns}^{-/-}$mice, leading to renal tubular cell damage and formation of cystine crystals. On the other hand, the elevated accumulation of cystine was clearly observed in both LEA/Tohm (3.9-fold) and F344-Ctns ${ }^{u g l}$ kidney (4.3fold) compared to age-matched control strains, and cystine contents in the kidneys of these strains were similar (LEA/ Tohm, $5.60 \pm 1.67$ vs. F344-Ctns ${ }^{u g / u g l l}, 6.68 \pm 0.38 \mu \mathrm{M}$ of half-cystine/g of wet tissue; $P=0.28$ ) (Table 3; Fig. 2c). Based on these findings, it is unlikely that the early onset of glucosuria observed in LEA/Tohm rats is correlated with the amount of cystine accumulation in renal tissues. It is possible that impaired glucose tolerance (Okamura et al. 2013), 


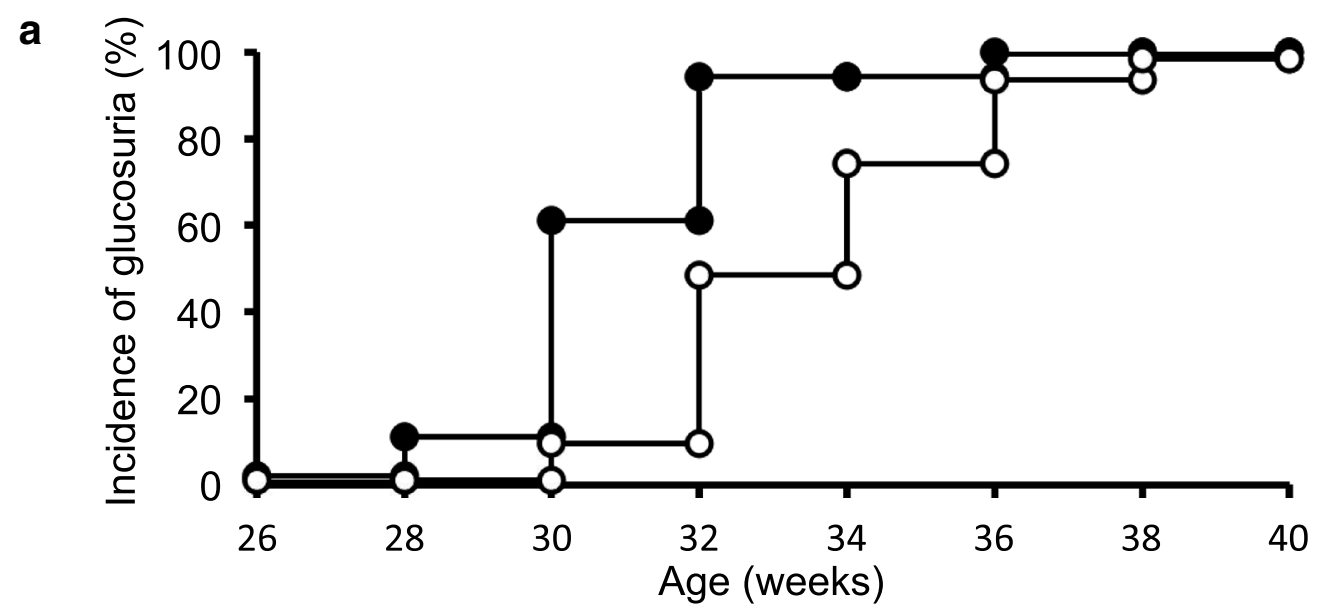

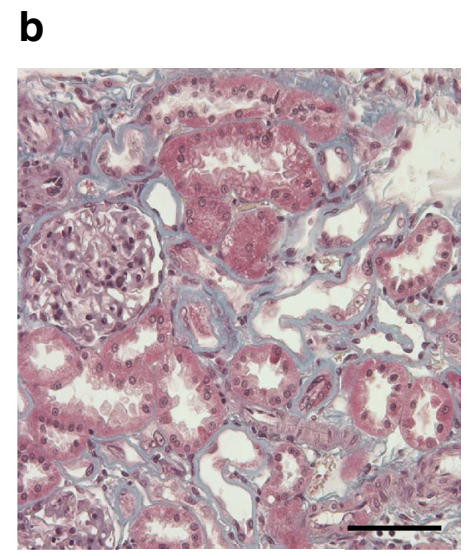

LEA/Tohm

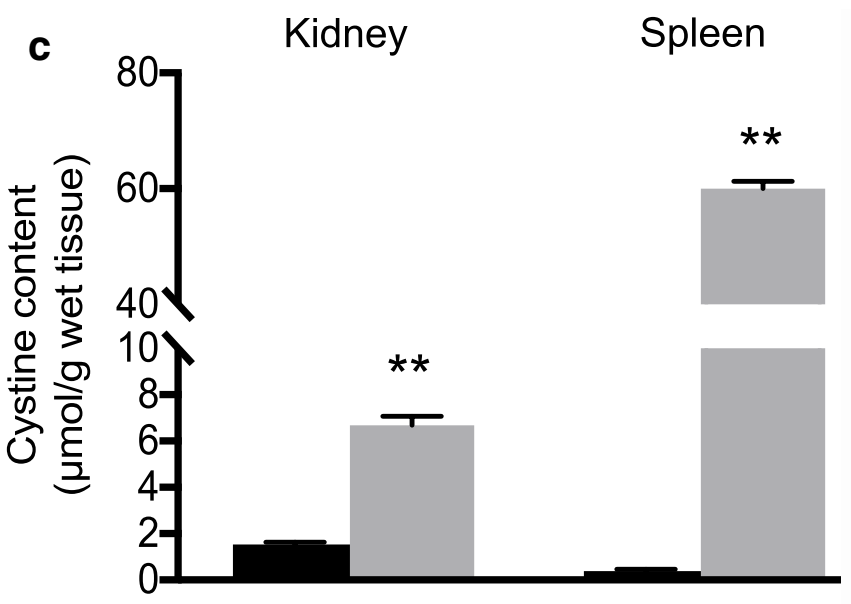

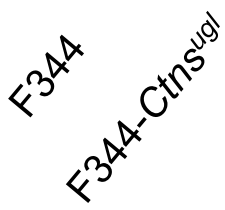

F344-Ctns ${ }^{u g l}$

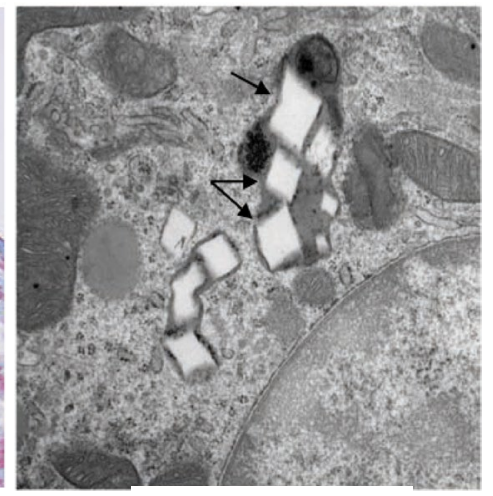

F344-Ctns ${ }^{u g l}$

Bone marrow

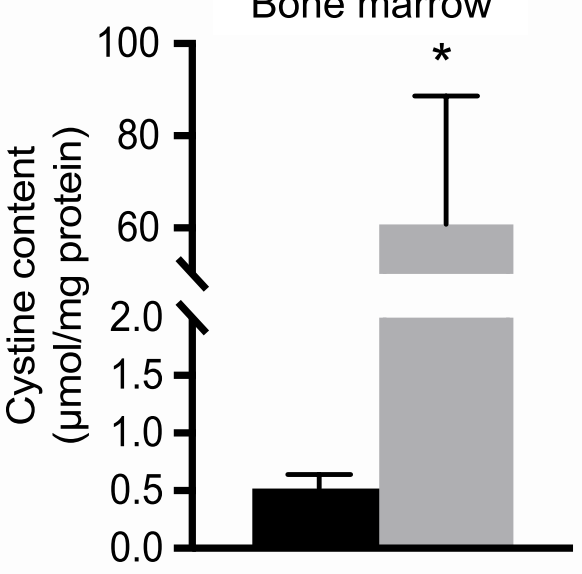

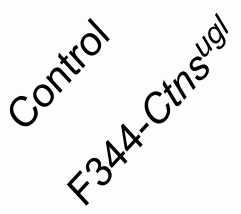


4Fig. 2 Phenotypic analysis of F344-Ctns ${ }^{u g l}$ rats. a The cumulative incidence of glucosuria in F344-Ctns ${ }^{u g l}$ male (closed circle; $n=18$ ) and female (open circle; $n=31$ ) rats. Glucosuria is defined as urine glucose levels of $50 \mathrm{mg} / \mathrm{dL}$ or higher under fasting conditions. b Renal histological section of male LEA/Tohm at 48 weeks of age (left panel) and male F344-Ctns ${ }^{u g l}$ rats at 52 weeks of age (middle panel) stained with Masson's Trichrome. Scale bar $=100 \mu \mathrm{m}$. Right panel: Representative TEM image of male F344-Ctns ${ }^{u g l}$ kidney cortex at 52 weeks of age. Arrows indicate cystine crystals in lysosomes. Magnification $\times 25,000$. $\mathbf{c}$ Cystine contents of kidney and spleen (left panel) at 38-42 weeks of age, and of bone marrow (right panel) at 40 weeks of age in male F344 ( $n=3$; black bars) rats and male F344-Ctns ${ }^{\text {ugl }}$ ( $n=3$; gray bars) rats. $* P<0.05$, $* * P<0.01$

which may result in glucotoxicity in the renal tubular cells, accelerated the onset of renal tubulopathy.

The cystine assay is the cornerstone in the treatment of cystinosis, both for diagnosis and therapeutic monitoring of the disease. HPLC is frequently used to detect amino acids and provides more cost-effective and easy-to-use methods compared with LC/MS/MS. Our cystine detection method using HPLC with a metal-free column successfully measured the cystine levels in various tissues obtained from control and cystinosis model rats (Table 3; Fig. 2c). Since this column does not contain metals and there was a low risk of contamination with metal impurities, we concluded that this column is more suitable for the analysis of cystine, which is the oxidized form of cysteine, and has a disulfide bond, a site of the redox reaction. However, it was difficult to measure the cystine content in the leukocytes separated from $3 \mathrm{~mL}$ of blood from the control strain rats using HPLC, although this content was detectable in leukocytes of F344-Ctns $s^{u g l / u g l}$ rats. Our cystine analysis method using HPLC is sufficient for quantifying the cystine content in cystine-accumulated tissues and leukocytes, but higher sensitivity analysis using LC/MS/MS is required for detecting the low levels of cystine in the leukocytes of healthy controls (Chabli et al. 2007; Garcia-Villoria et al. 2013).

Cysteamine, a simple aminothiol molecule, is the endogenous reducing agent and intermediate product of the taurine biosynthesis pathway. Several clinical trials have indicated that the reduction of cystine levels in cystinosis patients treated with oral cysteamine is critical to delay the disease progression and to prolong life (Brodin-Sartorius et al. 2012; Gahl et al. 2002). In cystinosis cells treated with cysteamine, the drug enters the lysosome through an unidentified transporter where it breaks the disulfide bond in cystine, leading to the formation of cysteine and cysteine-cysteamine disulfide. Cysteine and cysteine-cysteamine disulfide can leave the lysosome via cysteine and an unidentified cationic amino acid transporter, respectively, resulting in a marked reduction of cystine in the cells (Besouw et al. 2013). However, the adverse effects, which include anorexia, vomiting, unpleasant odor, and halitosis, often lead to poor compliance with oral cysteamine therapy. Additionally, cysteamine stimulates gastric acid secretion, leading to gastrointestinal ulceration when administered to rats in high doses (Selye and Szabo 1973). Therefore, the development of new therapies, such as hematopoietic stem cell transplantation (Gaide Chevronnay et al. 2016; Syres et al. 2009; Yeagy et al. 2011) and other oral medicines, is expected to improve the prognosis of cystinosis patients. We compared the effect of captopril [a specific competitive inhibitor of angiotensin I-converting enzyme (ACE)] and bucillamine (a cysteine derivative) on the reduction of intracellular cystine content using F344-Ctns ${ }^{\text {ugl }}{ }^{\text {ugl }}$ REFs (Fig. 3). Captopril is a sulfhydryl ACE inhibitor initially used in the treatment of hypertension and heart failure, and is protective against retinopathy and nephropathy (Parving et al. 1988; UK Prospective Diabetes Study Group 1998). Bucillamine is a homolog of D-penicillamine with two thiol groups, widely used to treat rheumatoid arthritis in Japan (Sagawa et al. 2011). Treatment with captopril or bucillamine decreased intracellular cystine in a dose-dependent manner. However, treatment with captopril and bucillamine less effectively reduced the intracellular cystine levels than that with cysteamine (Fig. 3a, b). These results indicate that REFs from F344$C_{\text {tns }}{ }^{\text {ugl } / \text { ugl }}$ rats are a valuable tool to evaluate and develop new cystine depleting treatments.

Oral cysteamine treatment delays disease progression and improves the life expectancy of cystinosis patients, but ultimately cannot eliminate the disease. We identified the $C_{t n s}{ }^{u g l}$ mutation, which leads to cystine accumulation in various tissues and results in renal tubulopathy, and developed a novel congenic rat strain harboring the $C t n s^{u g l}$ mutation. Although further biochemical and pathophysiological analyses of F344-Ctns ${ }^{u g l}$ rats are needed to evaluate their usefulness as a model of cystinosis, the evidence provided in the present study suggests that F344-Ctns ${ }^{u g l}$ rats will be an invaluable tool to expand our understanding of cystinosis pathogenesis, and for developing and testing novel therapeutic treatments.

Acknowledgements We thank Prof. T. Sakai (Juntendo University) and the late Prof. T. Kanai (Tokyo Women's Medical University) for insightful comments and suggestions, and Dr. M. Tamura-Nakano (NCGM) for technical assistance with electron microscopy. We also thank to the National BioResource Project-Rat (NBRP-Rat) for providing the rat genome DNAs. This study was supported by Grants-in Aid for Research from the National Center for Global Health and Medicine (27-6001 and 29-2004) and partially supported by Grant-in-Aid for Scientific Research, KAKENHI (17K07149) from the Ministry of Education, Culture, Sports, Science and Technology (MEXT). 

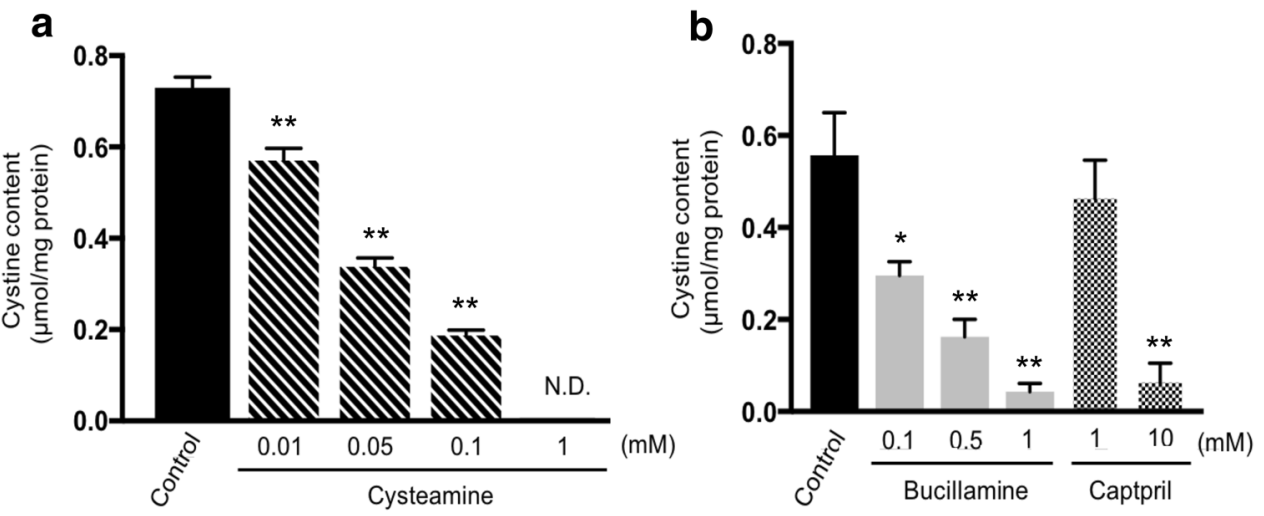

Fig. 3 Cystine contents of F344-Ctns ${ }^{\text {ugl }}$ REFs treated with drugs containing thiol groups: a cysteamine (hatched bars), b bucillamine (gray bars), and captopril (dotted bars). REFs were incubated in culture medium containing each drug for $24 \mathrm{~h}$. Control cells (black bar) were

\section{Compliance with ethical standards}

Conflict of interest The authors declare no conflict of interest.

Ethical approval All animal experiments were approved by the President of NCGM, following consideration by the Institutional Animal Care and Use Committee of NCGM (Approval ID: No. 17055), and were conducted in accordance with institutional procedures, national guidelines and the relevant national laws on the protection of animals.

Open Access This article is distributed under the terms of the Creative Commons Attribution 4.0 International License (http://creativeco mmons.org/licenses/by/4.0/), which permits unrestricted use, distribution, and reproduction in any medium, provided you give appropriate credit to the original author(s) and the source, provide a link to the Creative Commons license, and indicate if changes were made.

\section{References}

Agui T, Miyamoto T, Tsumagari T (2001) X-ray hypersensitivity in the LEA rat: genetic linkage analysis of responsible loci. Exp Anim 50:147-151

Attard M, Jean G, Forestier L, Cherqui S, van't Hoff W, Broyer M, Antignac C, Town M (1999) Severity of phenotype in cystinosis varies with mutations in the CTNS gene: predicted effect on the model of cystinosin. Hum Mol Genet 8:2507-2514

Besouw M, Masereeuw R, van den Heuvel L, Levtchenko E (2013) Cysteamine: an old drug with new potential. Drug Discov Today 18:785-792

Brodin-Sartorius A, Tete MJ, Niaudet P, Antignac C, Guest G, Ottolenghi C, Charbit M, Moyse D, Legendre C, Lesavre P, Cochat P, Servais A (2012) Cysteamine therapy delays the progression of nephropathic cystinosis in late adolescents and adults. Kidney Int 81:179-189

Chabli A, Aupetit J, Raehm M, Ricquier D, Chadefaux-Vekemans B (2007) Measurement of cystine in granulocytes using liquid chromatography-tandem mass spectrometry. Clin Biochem 40:692-698 cultured under the same conditions, with the addition of REF medium in place of the drug. $* P<0.05, * * P<0.01$ versus control. N.D. signifies "not detected"

Cherqui S (2012) Cysteamine therapy: a treatment for cystinosis, not a cure. Kidney Int 81:127-129

Chuang YW, Wen MC, Wu MJ, Shu KH, Cheng CH, Yu TM, Huang ST, Chen CH (2012) Follow-up and treatment of renal transplantation with nephropathic cystinosis in central Taiwan. Transpl Proc 44:80-82

Elmonem MA, Veys KR, Soliman NA, van Dyck M, van den Heuvel LP, Levtchenko E (2016) Cystinosis: a review. Orphanet J Rare Dis. https://doi.org/10.1186/s13023-016-0426-y

Gahl WA (1986) Cystinosis coming of age. Adv Pediatr 33:95-126

Gahl WA, Thoene JG, Schneider JA (2002) Cystinosis. N Engl J Med 347:111-121

Gahl WA, Balog JZ, Kleta R (2007) Nephropathic cystinosis in adults: natural history and effects of oral cysteamine therapy. Ann Intern Med 147:242-250

Gaide Chevronnay HP, Janssens V, Van Der Smissen P, Rocca CJ, Liao XH, Refetoff S, Pierreux CE, Cherqui S, Courtoy PJ (2016) Hematopoietic stem cells transplantation can normalize thyroid function in a cystinosis mouse model. Endocrinology 157:1363-1371

Garcia-Villoria J, Hernandez-Perez JM, Arias A, Ribes A (2013) Improvement of the cystine measurement in granulocytes by liquid chromatograhy-tandem mass spectrometry. Clin Biochem 46:271-274

Hamase K (2007) Sensitive two-dimensional determination of small amounts of D-amino acids in mammals and the study on their functions. Chem Pharm Bull 55:503-510

Higashi S, Matsunoshita N, Otani M, Tokuhiro E, Nozu K, Ito S (2017) Diagnostic challenge in a patient with nephropathic juvenile cystinosis: a case report. BMC Nephrol. https://doi. org/10.1186/s12882-017-0721-4

Hirokawa T, Boon-Chieng S, Mitaku S (1998) SOSUI: classification and secondary structure prediction system for membrane proteins. Bioinformatics 14:378-379

Iglesias DM, El-Kares R, Taranta A, Bellomo F, Emma F, Besouw M, Levtchenko E, Toelen J, van den Heuvel L, Chu L, Zhao J, Young YK, Eliopoulos N, Goodyer P (2012) Stem cell microvesicles transfer cystinosin to human cystinotic cells and reduce cystine accumulation in vitro. PLoS ONE 7:e42840

Kalatzis V, Cherqui S, Antignac C, Gasnier B (2001) Cystinosin, the protein defective in cystinosis, is a $\mathrm{H}(+)$-driven lysosomal cystine transporter. EMBO J 20:5940-5949 
Kalatzis V, Nevo N, Cherqui S, Gasnier B, Antignac C (2004) Molecular pathogenesis of cystinosis: effect of CTNS mutations on the transport activity and subcellular localization of cystinosin. Hum Mol Genet 13:1361-1371

Konno R, Okamura T, Kasai N, Summer KH, Niwa A (2009) Mutant rat strain lacking D-amino-acid oxidase. Amino Acids 37:367-375

Markel P, Shu P, Ebeling C, Carlson GA, Nagle DL, Smutko JS, Moore KJ (1997) Theoretical and empirical issues for marker-assisted breeding of congenic mouse strains. Nat Genet 17:280-284

Masuyama T, Fuse M, Yokoi N, Shinohara M, Tsujii H, Kanazawa M, Kanazawa Y, Komeda K, Taniguchi K (2003) Genetic analysis for diabetes in a new rat model of nonobese type 2 diabetes, Spontaneously Diabetic Torii rat. Biochem Biophys Res Commun 304:196-206

Nakajima M, Kato J, Kohgo Y, Takeichi N, Niitsu T (1994) Point mutation of aldehyde dehydrogenase-2 gene in mutant strains of LongEvans rats. Alcohol Alcohol Suppl 29:39-43

Nakano K, Yanobu-Takanashi R, Takahashi Y, Sasaki H, Shimizu Y, Okamura T, Sasaki N (2018) Novel murine model of congenital diabetes: the insulin hyposecretion mouse. J Diabetes Investig. https://doi.org/10.1111/jdi.12895

Nevo N, Chol M, Bailleux A, Kalatzis V, Morisset L, Devuyst O, Gubler MC, Antignac C (2010) Renal phenotype of the cystinosis mouse model is dependent upon genetic background. Nephrol Dial Transplant 25:1059-1066

Okamura T, Pei XY, Miyoshi I, Shimizu Y, Takanashi-Yanobu R, Mototani Y, Kanai T, Satoh J, Kimura N, Kasai N (2013) Phenotypic characterization of LEA rat: a new rat model of nonobese type 2 diabetes. J Diabetes Res. https://doi.org/10.1155/2013/986462

Parving HH, Hommel E, Smidt UM (1988) Protection of kidney function and decrease in albuminuria by captopril in insulin dependent diabetics with nephropathy. BMJ 297:1086-1091

Piraud M, Vianey-Saban C, Petritis K, Elfakir C, Steghens JP, Morla A, Bouchu D (2003) ESI-MS/MS analysis of underivatised amino acids: a new tool for the diagnosis of inherited disorders of amino acid metabolism. Fragmentation study of 79 molecules of biological interest in positive and negative ionisation mode. Rapid Commun Mass Spectrom 17:1297-1311

Prencipe G, Caiello I, Cherqui S, Whisenant T, Petrini S, Emma F, De Benedetti F (2014) Inflammasome activation by cystine crystals: implications for the pathogenesis of cystinosis. J Am Soc Nephrol 25:1163-1169

Sagawa A, Fujisaku A, Ohnishi K, Mukai M, Yasuda I, Amasaki Y, Shimizu M, Ichikawa K, Ohsaki H (2011) A multicentre trial of bucillamine in the treatment of early rheumatoid arthritis (SNOW study). Mod Rheumatol 21:251-257
Sansanwal P, Yen B, Gahl WA, Ma Y, Ying L, Wong LJ, Sarwal MM (2010) Mitochondrial autophagy promotes cellular injury in nephropathic cystinosis. J Am Soc Nephrol 21:272-283

Sasaki N, Hayashizaki Y, Muramatsu M, Matsuda Y, Ando Y, Kuramoto T, Serikawa T, Azuma T, Naito A, Agui T, Yamashita T, Miyoshi I, Takeichi N, Kasai N (1994) The gene responsible for LEC hepatitis, located on rat chromosome 16, is the homolog to the human Wilson disease gene. Biochem Biophys Res Commun 202:512-518

Selye H, Szabo S (1973) Experimental model for production of perforating duodenal ulcers by cysteamine in the rat. Nature 244:458-459

Syres K, Harrison F, Tadlock M, Jester JV, Simpson J, Roy S, Salomon DR, Cherqui S (2009) Successful treatment of the murine model of cystinosis using bone marrow cell transplantation. Blood 114:2542-2552

Touchman JW, Anikster Y, Dietrich NL, Maduro VV, McDowell G, Shotelersuk V, Bouffard GG, Beckstrom-Sternberg SM, Gahl WA, Green ED (2000) The genomic region encompassing the nephropathic cystinosis gene (CTNS): complete sequencing of a $200-\mathrm{kb}$ segment and discovery of a novel gene within the common cystinosis-causing deletion. Genome Res 10:165-173

Town M, Jean G, Cherqui S, Attard M, Forestier L, Whitmore SA, Callen DF, Gribouval O, Broyer M, Bates GP, van't Hoff W, Antignac C (1998) A novel gene encoding an integral membrane protein is mutated in nephropathic cystinosis. Nat Genet 18:319-324

UK Prospective Diabetes Study Group (1998) Tight blood pressure control and risk of macrovascular and microvascular complications in type 2 diabetes: UKPDS 38. BMJ 317:703-713

Vitvitsky V, Witcher M, Banerjee R, Thoene J (2010) The redox status of cystinotic fibroblasts. Mol Genet Metab 99:384-388

Yang YJ, Hu Y, Zhao R, He X, Zhao L, Tu M, Zhou L, Guo J, Wu L, Zhao T, Zhu YM (2015) First report of CTNS mutations in a Chinese family with infantile cystinosis. Sci World J. https://doi. org/10.1155/2015/309410

Yeagy BA, Harrison F, Gubler MC, Koziol JA, Salomon DR, Cherqui S (2011) Kidney preservation by bone marrow cell transplantation in hereditary nephropathy. Kidney Int 79:1198-1206

Publisher's Note Springer Nature remains neutral with regard to jurisdictional claims in published maps and institutional affiliations. 